\title{
Synthesis of Histone Deacetylases Inhibitor and Activity in Vitro
}

\author{
Baoping KUANG ${ }^{1, a}$, Hui ZHANG ${ }^{2, b}$, Zhihang SHANG ${ }^{3, c}$ \\ ${ }^{1}$ Department of Physics, Shenyang Medical College, Shenyang, 110034, China \\ 2 Department of Chemistry, Shenyang Medical College, Shenyang, 110034, China \\ ${ }^{3}$ The center of Functional Experiment, Shenyang Medical College, Shenyang, 110034, China \\ a email: kbpzyky@163.com, bemail:a13998826073@163.com, 'email:a13940089073@163.com
}

\section{Keywords: Vorinostat; Histone Deacetylases Inhibitor; Multi-Drug Resistance}

\begin{abstract}
Multi-drug resistance (MDR) is an important element which leads to ineffectiveness of chemotherapeutics. The discovery of histone acetylase's unusual activities in tumor cells provides a new area for the cure of cancer. And histone deacetylases inhibitor (HDACi) is used as a new agent in clinical therapy. New histone deacetylases inhibitors have been synthesized. All the products have been characterized by ${ }^{1} \mathrm{H}-\mathrm{NMR}$ and MS. The activities in vitro effects on the title compounds were examined. From the biological activity results, we found that three compounds showed good inhibitory potency against HDACs and anti-proliferate activities to inhibit human colonic cancer cells growth.Introduction.
\end{abstract}

\section{Introduction}

Vorinostat (SAHA) is a kind of histone deacetlyases inhibitors anticancer drug[1]. Histone deacetylases inhibitors are a group of compound regulating gene expression at transcriptional level. The histone deacetylases inhibitors are potent inducers of growth arrest, differentiation and apoptosis of tumor cells. Vorinostat contains this function [2, 3].

In tumor cells, histone deacetylases (HDACs) are over-expressed resulting in excessive deactivated his tones which packed DNA tightly to form an abnormal "compact structure" of chromatin. Inhibition of HDACs could potently inhibit proliferation of tumor cells, cell cycle arrest and apoptosis, which makes HDACs become epigenetic targets for designing anti- cancer agents. As a result, HDACi has been recognized as an effectual strategy for cancer therapy [4, 5].

Small molecular hydroxirnate HDACs inhibitors (HDACi) have achieved significant biological effects in preclinical models of cancer and evoke popularity [6, 7]. In October 2006, the first hydroximate HDACi drug- SAHA (General name: Vorinostat, Trade name: Zolinza) was approved by FDA for treatment of T-cell lymphoma. Nowadays, more and more hydroximate HDACi entered clinical trials for treatment of solid tumor and hematological malignancy [8, 9].

In recent years[10, 11], there has been an effort to develop HDACs for cancer therapy, and Vorinostat (SAHA) has been approved for treatment of cutaneous $\mathrm{T}$ cell lymphoma (CTCL) in October 2006. The exact mechanisms by which the compounds may work are unclear, but epigenetic pathways are proposed.SAHA is proposed as a novel, but less toxic, anticancer therapy with little multidrug-resistance. So we synthesized SAHA and a series of new compounds, identify their structure and compare their IC $_{50}$ to SAHA's, attempting to open a new chapter for cancer chemotheraputics.

Here comes the design and synthesis of novel class of HDACi. In this research, HDACi was used as target and the hydroximate HDACi with the scaffold of cinnamamide containing excellent antineoplastic activity both in vitro was used as lead compound[12]. A novel series of cinnamamide hydroximates were designed and synthesized by means of isosteric principle[13]. Totally 20 new compounds were synthesized through reactions of esterification, Williamson reaction, specification and condensation. 


\section{Results and Discussion}
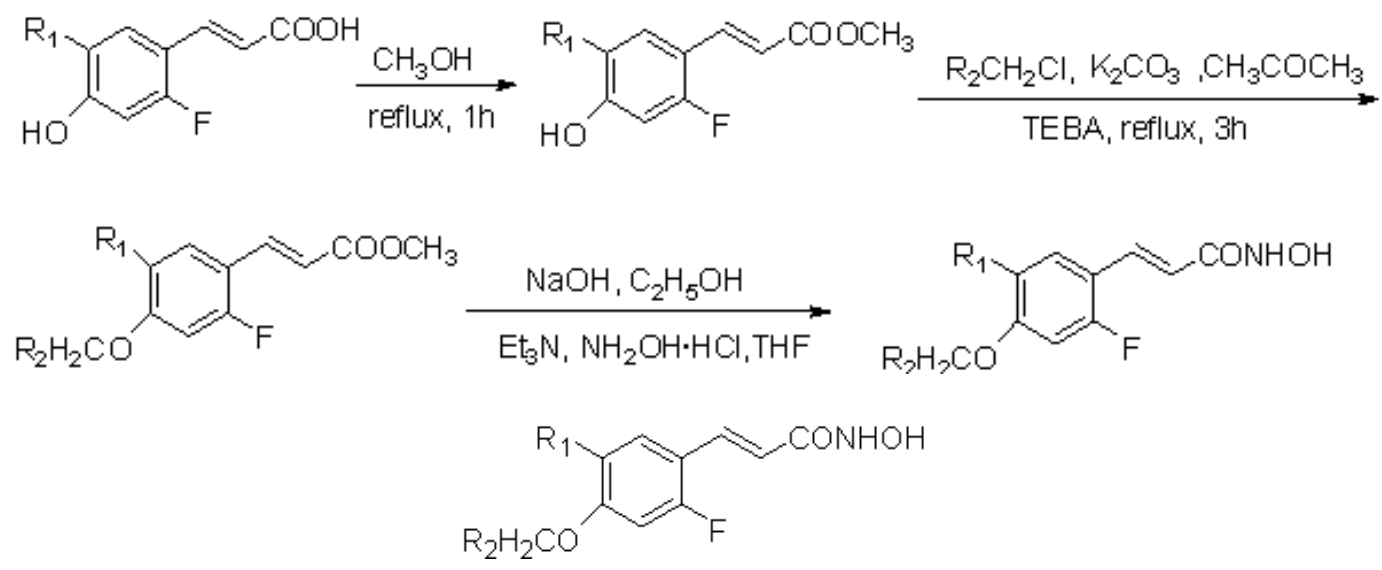

Scheme 1. Synthesis of the title compounds 1-20

Table 1. The Title Effect on Activity in Vitro

\begin{tabular}{|c|c|c|c|c|c|c|c|}
\hline Compd & $\mathrm{R}_{1}$ & $\mathrm{R}_{2}$ & $\begin{array}{l}\text { HDACs } \\
\mathrm{IC}_{50}(\mu \mathrm{M})\end{array}$ & Compd & $\mathrm{R}_{1}$ & $\mathrm{R}_{2}$ & $\begin{array}{l}\text { HDACs } \\
\mathrm{IC}_{50}(\mu \mathrm{M})\end{array}$ \\
\hline 1 & $\mathrm{H}$ & & 18.8 & 11 & $\mathrm{H}$ & & 45.2 \\
\hline 2 & $\mathrm{OCH}_{3}$ & & 13.0 & 12 & $\mathrm{OCH}_{3}$ & & 29.0 \\
\hline 3 & $\mathrm{H}$ & & 11.5 & 13 & $\mathrm{H}$ & & 6.5 \\
\hline 4 & $\mathrm{OCH}_{3}$ & & 10.8 & 14 & $\mathrm{OCH}_{3}$ & & 3.2 \\
\hline 5 & $\mathrm{H}$ & & 28.8 & 15 & $\mathrm{H}$ & & 10.6 \\
\hline 6 & $\mathrm{OCH}_{3}$ & & 20.6 & 16 & $\mathrm{OCH}_{3}$ & & 18.5 \\
\hline 7 & $\mathrm{H}$ & & 22.5 & 17 & $\mathrm{OCH}_{3}$ & & 28.2 \\
\hline 8 & $\mathrm{OCH}_{3}$ & & 12.8 & 18 & $\mathrm{OCH}_{3}$ & & 82.3 \\
\hline 9 & $\mathrm{H}$ & & 11.2 & 19 & $\mathrm{OCH}_{3}$ & & 55.3 \\
\hline 10 & $\mathrm{OCH}_{3}$ & & 2.2 & 20 & $\mathrm{OCH}_{3}$ & & 76.8 \\
\hline SAHA & & & & & & & 1.6 \\
\hline
\end{tabular}

Table 1 shows us inhibitory activities of the 20 target compounds to HDACS in vitro. As we can see the $\mathrm{IC}_{50}$ of the all new compounds we syntheized are higher than SAHA's IC $_{50}$ which is only 1.6. But most of their $\mathrm{IC}_{50}$ is lower than $30 \mu \mathrm{M}$. Especially, compound 10, 13, 14 have relative lower $\mathrm{IC}_{50}$ which are 2.2, 6.5 and 3.2 respectively. So we can say that the inhibitory activities of compound 10, 13 and 14 are relatively higher than other compounds but are a little lower than SAHA. 


\section{SAR analysis.}

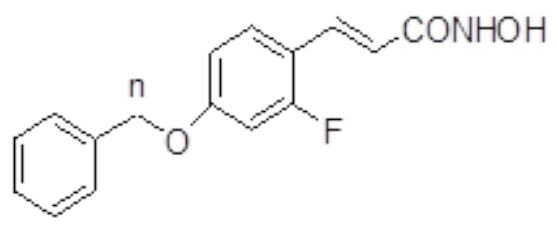

(1) The lower activity with $n$, when $n=1$ the activity is better.

(2) Methoxy $\left(\mathrm{CH}_{3} \mathrm{O}\right)$ group SAR:

The HDAC inhibition activities of compounds with methoxy group $\left(\mathrm{CH}_{3} \mathrm{O}\right)$ are higher than that of compounds with hydrogen $(\mathrm{H})$. HDAC inhibitory activities of most compounds with methoxy group are higher compounds with no methoxy group. It is prompted that methoxy group $\left(\mathrm{CH}_{3} \mathrm{O}\right)$ was introduced to benzene ring, we would get better inhibitors.

(3) 3-Pyridyl was replaced by phenyl group so that the activities are lower. 2- Thienyl or 2- furyl was replaced by phenyl group so that the activities are higher.

(4) The Aromatic Ring SAR:

Positioning a chlorine at the para position had little effect relative to the parent compound on the inhibition of HDAC, fluorin and methoxy group was better effect.

The activities relatively are as follower:

$\mathrm{R}^{\prime}$ are methoxy group $\left(\mathrm{CH}_{3} \mathrm{O}\right)$ and fluorin $(\mathrm{F})>$ methyl $\left(\mathrm{CH}_{3}\right)=$ hydrogen $(\mathrm{H}) \quad>$ chlorine $(\mathrm{Cl})>$ ethyl $\left(\mathrm{CH}_{3} \mathrm{CH}_{2}\right)$

Bioactivity against cancer cell lines.

Compound 4, 10 and 14 are selected to detect their inhibitory activity effect on colon cancer HCT116 cell. HCT116 cell has highest expression in tumor cell line, being used as the preferred tumor cell line to screen the activity of histone deacetylases inhibitor and A549 cell has relatively low expression. We used these tumor cell lines to test the activity of antitumor of the compounds. The result is as followings.

Table 2 Inhibitory Activity Effect on Cell Proliferation in Vitro

\begin{tabular}{|c|c|c|c|c|c|}
\hline \multirow[b]{2}{*}{ Compd.. } & \multicolumn{4}{|c|}{ Inhibition (\%) } & \multirow[t]{2}{*}{$\begin{array}{l}\text { HCT116 } \\
\text { IC }_{50}(\mu \mathrm{M})\end{array}$} \\
\hline & $400 \mu \mathrm{g} / \mathrm{mL}$ & $200 \mu \mathrm{g} / \mathrm{mL}$ & $100 \mu \mathrm{g} / \mathrm{mL}$ & $50 \mu \mathrm{g} / \mathrm{mL}$ & \\
\hline 4 & 92.5 & 91.2 & 84.7 & 75.6 & 84 \\
\hline 10 & 94.5 & 82.2 & 57.8 & 23.1 & 261 \\
\hline 14 & 98.1 & 92.5 & 75.9 & 43.5 & 148 \\
\hline SAHA & 79.8 & 77.0 & 72.6 & 50.6 & 148 \\
\hline \multicolumn{6}{|c|}{ Table 3 Inhibitory Activity Effect on Cell Proliferation in Vitro } \\
\hline & \multicolumn{4}{|c|}{ Inhibition (\%) } & $\begin{array}{c}\mathrm{A} 549 \\
\mathrm{IC}_{50}(\mu \mathrm{M})\end{array}$ \\
\hline Compd.. & $400 \mu \mathrm{g} / \mathrm{mL}$ & $200 \mu \mathrm{g} / \mathrm{mL}$ & $100 \mu \mathrm{g} / \mathrm{mL}$ & $50 \mu \mathrm{g} / \mathrm{mL}$ & \\
\hline 4 & 60.4 & 57.0 & 38.1 & 29.4 & 344 \\
\hline 10 & 25.0 & 13.4 & - & - & $>1000$ \\
\hline 14 & 54.8 & 31.3 & 15.1 & - & 578 \\
\hline SAHA & 54.9 & 49.4 & 37.6 & 23.2 & 583 \\
\hline
\end{tabular}


Table 2 and Table 3 shows that the inhibitory activity of compound $\mathbf{4}$ to these tumor cell's reproduction is the highest, which is higher than the positive control group such as SAHA and other compounds; the inhibitory activity of compound $\mathbf{1 4}$ is similar to that of SAHA; compound $\mathbf{1 0}$ has a higher inhibition activity to enzyme in vitro, but its inhibitory activity to tumor growth is much lower than that of SAHA.

\section{Experimental}

\section{General.}

Melting points for the compounds were determined on a hot-stage microscope and are uncorrected. ${ }^{1} \mathrm{H}-\mathrm{NMR}$ spectra were recorded in DMSO- $\mathrm{d}_{6}$ solution on a Bruker ARX-300 spectrometer operating at $300 \mathrm{MHz}$ with TMS as the internal reference. Coupling constants $(J)$ are expressed in Hz. MS spectra were obtained using a Finnigan SSQ-710 spectrometer. Column chromatography was performed on silica gel (200-300 mesh) obtained from Qingdao Ocean Chemicals. Unless otherwise noted, all the materials were obtained from commercial sources and used without further purification.

Preparation and analytical and spectral data for compounds 1-20.

Compd. (1): m.p. $175-176^{\circ} \mathrm{C}$ (from ethanol); ${ }^{1} \mathrm{H}-\mathrm{NMR} \delta$ : 5.25 (s, 2H), 6.32 (d, 1H, J=15.6), 7.06 (s, 2H), 7.42 (d, 1H, J=15.6), 7.31-7.42 (m, 6H), 8.96 (s, 1H), 10.67 (s, 1H). MS m/z (ESI): found for $\mathrm{C}_{16} \mathrm{O}_{3} \mathrm{NH}_{14} \mathrm{~F}[\mathrm{M}+\mathrm{H}] 288.43$.

Compd. (2): m.p. $169-170^{\circ} \mathrm{C}$ (from ethanol); ${ }^{1} \mathrm{H}-\mathrm{NMR} \delta: 3.81$ (s, 3H),5.13(s, 2H), 6.35(d, $1 \mathrm{H}$, $\mathrm{J}=15.6)$, 7.04-7.18 (m, 2H), 7.33-7.46 (m, 6H), 9.00 (s, 1H), 10.66 (s, 1H). MS m/z (ESI): found for $\mathrm{C}_{17} \mathrm{O}_{4} \mathrm{NH}_{16} \mathrm{~F}[\mathrm{M}+\mathrm{H}] 318.52$.

Compd. (3): m.p. $173-175^{\circ} \mathrm{C}$ (from ethanol); ${ }^{1} \mathrm{H}-\mathrm{NMR} \delta$ : 5.34(s, 2H), 6.35(d, 1H, J=15.6), 7.04-7.08 (m, 2H), 7.23(s, 1H), 7.42(d, 1H, J=15.6), 7.67-7.50(m, 3H), 9.00 (s, 1H), 10.68 (s, 1H). MS m/z (ESI): found for $\mathrm{C}_{14} \mathrm{O}_{3} \mathrm{NH}_{12} \mathrm{FS}[\mathrm{M}+\mathrm{H}] 294.38$.

Compd. (4): m.p. $151-153^{\circ} \mathrm{C}$ (from ethanol); ${ }^{1} \mathrm{H}-\mathrm{NMR} \delta$ : 3.88(s, 3H), 5.32(s, 2H), 6.39(d, $1 \mathrm{H}$, $\mathrm{J}=15.6), 7.02-7.10(\mathrm{~m}, 2 \mathrm{H}), 7.22(\mathrm{~s}, 1 \mathrm{H}), \quad 7.26(\mathrm{~s}, 1 \mathrm{H}), 7.41(\mathrm{~d}, 1 \mathrm{H}, \quad \mathrm{J}=15.6), 7.55(\mathrm{~s}, 1 \mathrm{H}), 9.00$ (s, $1 \mathrm{H}), 10.66(\mathrm{~s}, 1 \mathrm{H}) . \mathrm{MS} \mathrm{m} / \mathrm{z}(\mathrm{ESI})$ : found for $\mathrm{C}_{15} \mathrm{O}_{4} \mathrm{NH}_{14} \mathrm{FS}[\mathrm{M}+\mathrm{H}] 324.36$.

Compd. (5): m.p. $192-194^{\circ} \mathrm{C}$ (from ethanol); ${ }^{1} \mathrm{H}-\mathrm{NMR} \delta: 5.20(\mathrm{~s}, 2 \mathrm{H}), 6.36(\mathrm{~d}, 1 \mathrm{H}, \mathrm{J}=15.6), 7.08$ (s, 2H), 7.40-7.56 (m, 3H), 7.88(d, 1H, J=7.8), 8.55(d, 1H, J=6.0), 8.65(s, 1H), 9.07 (s, 1H), 10.66 (s, 1H). MS m/z (ESI): found for $\mathrm{C}_{15} \mathrm{O}_{3} \mathrm{~N}_{2} \mathrm{H}_{13} \mathrm{~F}[\mathrm{M}+\mathrm{H}] 289.32$.

Compd. (6): m.p. $178-179^{\circ} \mathrm{C}$ (from ethanol); ${ }^{1} \mathrm{H}-\mathrm{NMR} \delta$ : 3.83(s, 6H), 5.20(s, 2H), 6.36(d, $1 \mathrm{H}$, $\mathrm{J}=15.6)$, 7.02-7.20 (m, 3H), 7.33-7.53 (m, 3H), 7.84(d, 1H, J=7.8), 8.56(s, 1H), 9.02 (s, 1H), $10.66(\mathrm{~s}, 1 \mathrm{H})$. MS m/z (ESI): found for $\mathrm{C}_{16} \mathrm{O}_{4} \mathrm{~N}_{2} \mathrm{H}_{15} \mathrm{~F}[\mathrm{M}+\mathrm{H}] 319.36$.

Compd. (7): m.p. $176-178^{\circ} \mathrm{C}$ (from ethanol); ${ }^{1} \mathrm{H}-\mathrm{NMR} \delta$ : 2.32(s, 3H), 5.10(s, 2H), 6.32(d, $1 \mathrm{H}$, $\mathrm{J}=15.6), 7.02-7.22$ (t, 3H), 7.32-7.50 (m, 5H), 8.99 (s, 1H), 10.66 (s, 1H). MS m/z (ESI): found for $\mathrm{C}_{17} \mathrm{O}_{3} \mathrm{NH}_{16} \mathrm{~F}[\mathrm{M}+\mathrm{H}] 302.30$.

Compd. (8): m.p. $173-175^{\circ} \mathrm{C}$ (from ethanol); ${ }^{1} \mathrm{H}-\mathrm{NMR} \delta: 2.32(\mathrm{~s}, 3 \mathrm{H}), 3.80$ (s, 3H), 5.08(s, 2H), 6.32(d, 1H, J=15.6), 7.02-7.18 (m, 3H), 7.18-7.34 (t, 3H), 7.42(d, 1H), 8.99 (s, 1H), 10.66 (s, 1H). MS m/z (ESI): found for $\mathrm{C}_{18} \mathrm{O}_{4} \mathrm{NH}_{18} \mathrm{~F}[\mathrm{M}+\mathrm{H}] 332.30$. $\quad \mathrm{C}_{18} \mathrm{O}_{4} \mathrm{NH}_{18} \mathrm{~F}$

Compd. (9): m.p. $184-186^{\circ} \mathrm{C}$ (from ethanol); ${ }^{1} \mathrm{H}-\mathrm{NMR} \delta: 3.76$ (s, 3H), 5.05(s, 2H), 6.31(d, 1H, $\mathrm{J}=15.6), 6.98-7.02(\mathrm{t}, 3 \mathrm{H}), 7.38-7.64(\mathrm{~m}, 5 \mathrm{H}), 8.99$ (s, 1H), 10.66 (s, 1H). MS m/z (ESI): found for $\mathrm{C}_{17} \mathrm{O}_{4} \mathrm{NH}_{16} \mathrm{~F}[\mathrm{M}+\mathrm{H}] 318.35$. 
Compd. (10): m.p. $168-170^{\circ} \mathrm{C}$ (from ethanol); ${ }^{1} \mathrm{H}-\mathrm{NMR} \delta: 3.76$ (s, 3H), 3.78(s, 3H), 5.03(s, 2H), 6.31(d, 1H, J=15.6), 6.96-7.06 (t, 3H), 7.08-7.38 (m, 3H), 7.42(d, 1H, J=15.6), 8.99 (s, 1H), 10.65 (s, $1 \mathrm{H}) . \mathrm{MS} \mathrm{m} / \mathrm{z}$ (ESI): found for $\mathrm{C}_{18} \mathrm{O}_{5} \mathrm{NH}_{18} \mathrm{~F}[\mathrm{M}+\mathrm{H}] 348.42$.

Compd. (11): m.p. $170-172^{\circ} \mathrm{C}$ (from ethanol); ${ }^{1} \mathrm{H}-\mathrm{NMR} \delta$ : 5.13(s, 2H), 6.31(d, $\left.1 \mathrm{H}, \mathrm{J}=15.6\right)$, 7.02-7.21 (t, 3H), 7.38-7.52 (m, 5H), $9.04(\mathrm{~s}, 1 \mathrm{H}), 10.66$ (s, 1H). MS m/z (ESI): found for $\mathrm{C}_{16} \mathrm{O}_{3} \mathrm{NH}_{13} \mathrm{FCl}[\mathrm{M}+\mathrm{H}] 322.90$.

Compd. (12): m.p. $165-167^{\circ} \mathrm{C}$ (from ethanol); ${ }^{1} \mathrm{H}-\mathrm{NMR} \delta$ : 3.81 (s, 3H ) , 5.13(s, 2H), 6.33(d, $1 \mathrm{H}$, $\mathrm{J}=15.6), 7.02-7.18(\mathrm{~m}, 2 \mathrm{H}), 7.38-7.50(\mathrm{~m}, 5 \mathrm{H}), 9.01$ (s, 1H), 10.66 (s, 1H). MS m/z (ESI): found for $\mathrm{C}_{17} \mathrm{O}_{4} \mathrm{NH}_{15} \mathrm{FCl}[\mathrm{M}+\mathrm{H}] 352.90$.

Compd. (13): m.p. $180-182^{\circ} \mathrm{C}$ (from ethanol); ${ }^{1} \mathrm{H}-\mathrm{NMR} \quad \delta: 5.13(\mathrm{~s}, 2 \mathrm{H}), 6.31(\mathrm{~d}, 1 \mathrm{H}, \mathrm{J}=15.6)$, 7.02-7.21 (t, 3H), 7.30-7.46 (m, 5H), $9.04(\mathrm{~s}, 1 \mathrm{H}), 10.66$ (s, 1H). MS m/z (ESI): found for $\mathrm{C}_{16} \mathrm{O}_{3} \mathrm{NH}_{13} \mathrm{~F}_{2}[\mathrm{M}+\mathrm{H}] 306.40$.

Compd. (14): m.p. $172-174^{\circ} \mathrm{C}$ (from ethanol); ${ }^{1} \mathrm{H}-\mathrm{NMR} \delta: 3.81$ (s, 3H ) , 5.13(s, 2H), 6.33(d, $1 \mathrm{H}$, $\mathrm{J}=15.6), 6.82-7.00(\mathrm{~m}, 2 \mathrm{H}), 7.38-7.50(\mathrm{~m}, 5 \mathrm{H}), 9.01$ (s, 1H), 10.66 (s, 1H). MS m/z (ESI): found for $\mathrm{C}_{17} \mathrm{O}_{4} \mathrm{NH}_{15} \mathrm{~F}_{2}[\mathrm{M}+\mathrm{H}] 336.30$.

Compd. (15): m.p. $163-165^{\circ} \mathrm{C}$ (from ethanol); ${ }^{1} \mathrm{H}-\mathrm{NMR} \quad \delta: 5.34(\mathrm{~s}, 2 \mathrm{H}), 6.35(\mathrm{~d}, 1 \mathrm{H}, \mathrm{J}=15.6)$, 7.04-7.08 (m, 2H), 7.13(s, 1H), 7.32(d, 1H, J=15.6), 7.67-7.50(m, 3H), 9.00 (s, 1H), 10.68 (s, 1H). MS m/z (ESI): found for $\mathrm{C}_{14} \mathrm{O}_{4} \mathrm{NH}_{12} \mathrm{~F}[\mathrm{M}+\mathrm{H}] 278.30$.

Compd. (16): m.p. $158-160^{\circ} \mathrm{C}$ (from ethanol); ${ }^{1} \mathrm{H}-\mathrm{NMR} \delta$ : 3.77 (s, 3H ) , 5.06(s, 2H), 6.35(d, 1H, $\mathrm{J}=15.6), 6.60-6.47(\mathrm{~m}, 2 \mathrm{H}), 7.08-7.13(\mathrm{~m}, 2 \mathrm{H}), 7.16(\mathrm{~s}, 1 \mathrm{H}), 7.39(\mathrm{~d}, 1 \mathrm{H}, \mathrm{J}=15.6), 7.70(\mathrm{~s}, 1 \mathrm{H}), 8.98$ (s, $1 \mathrm{H}), 10.64$ (s, $1 \mathrm{H}) . \mathrm{MS} \mathrm{m} / \mathrm{z}(\mathrm{ESI})$ : found for $\mathrm{C}_{15} \mathrm{O}_{5} \mathrm{NH}_{14} \mathrm{~F}[\mathrm{M}+\mathrm{H}] 308.40$

Compd. (17): m.p. $180-181^{\circ} \mathrm{C}$ (from ethanol); ${ }^{1} \mathrm{H}-\mathrm{NMR} \delta$ : 3.05(t, 2H, J=6.9), 3.78 (s, 3H ), 4.20(t, $2 \mathrm{H}, \mathrm{J}=6.9$ ), 6.35(d, 1H, J=15.6), 7.00-7.40(m, 8H), 8.95 (s, 1H), 10.64 (s, 1H). MS m/z (ESI): found for $\mathrm{C}_{18} \mathrm{O}_{4} \mathrm{NH}_{18} \mathrm{~F}[\mathrm{M}+\mathrm{H}] 332.40$.

Compd. (18): m.p. $175-177^{\circ} \mathrm{C}$ (from ethanol); ${ }^{1} \mathrm{H}-\mathrm{NMR} \delta: 3.05$ (t, 2H, J=6.9), 3.78 (s, 3H ), 4.20(t, 2H, J=6.9), 6.35(d, 1H, J=15.6), 7.00-7.40(m, 10H), 8.95 (s, 1H), 10.64 (s, 1H). MS m/z (ESI): found for $\mathrm{C}_{19} \mathrm{O}_{4} \mathrm{NH}_{20} \mathrm{~F}[\mathrm{M}+\mathrm{H}] 346.40$

Compd. (19): m.p. $134-139^{\circ} \mathrm{C}\left(\right.$ from ethanol); ${ }^{1} \mathrm{H}-\mathrm{NMR} \delta: 3.82$ (s, 3H) , 4.75(d, $2 \mathrm{H}, \mathrm{J}=5.7$ ), 6.35(d, 1H, J=15.6), 6.47-6.56(m, 3H), 6.76(d, 1H, J=15.9), 7.00-7.40(m, 6H), 8.99 (s, 1H), 10.66 (s, $1 \mathrm{H})$. MS m/z (ESI): found for $\mathrm{C}_{19} \mathrm{O}_{4} \mathrm{NH}_{18} \mathrm{~F}[\mathrm{M}+\mathrm{H}] 344.30$

Compd. (20): m.p. $160-162{ }^{\circ} \mathrm{C}$ (from ethanol); ${ }^{1} \mathrm{H}-\mathrm{NMR} \delta$ : 3.05(t, 2H, J=6.9), 3.78 (s, 3H ), 4.20(t, 2H, J=6.9), 6.35(d, 1H, J=15.6), 7.00-7.40(m, 10H), 8.95 (s, 1H), 10.64 (s, 1H). MS m/z (ESI): found for $\mathrm{C}_{19} \mathrm{O}_{4} \mathrm{NH}_{20} \mathrm{~F}[\mathrm{M}+\mathrm{H}] 346.42$.

\section{Conclusion}

In conclusion, a simple preparation of novel histone deacetylase inhibitors has been described. The key synthetic strategies involve diazotization, esterification, reduction, chloration with thionyl chloride and Williamson reactions carried out in general yields. Compounds 20 exhibited the some potent activity among these analogues. We have found that compound $\mathbf{4}$ and $\mathbf{1 0}$ showed growth inhibiting potency in vitro. Although all prepared compounds were exhibited less histone 
deacetylase (HDAC) inhibitors activity than SAHA as a compared material, our investigation suggests that hydroxamic acids, which have been explored extensively as HDAC inhibitors, are excellent for obtaining potent compounds.

\section{Acknowledgement}

At the end of this presentation I want to thank Prof. Li Pan for her technical assistance, Jian Tong for assistance with multidimensional NMR experiments, and providing coordinates of the published NMR structure of target compounds and high resolution mass spectral analyses. We acknowledge the fund program for a Researcher's Award of Shenyang Medical College. This work was supported by the department of chemical biology and pharmacology. Thanks a lot.

\section{References}

[1] Hubbs J.L.; Zhou H.; Kral A,M.; Fleming J.C.; Dahlberg W.K.; Hughes B.L.; Middleton R.E.; Szewczak A.A.; Secrist J.P.; Miller T.A. Amino acid derivatives as histone deacetylase inhibitors[J]. Bioorg Med Chem Lett, 2008, 18(1):34-8.

[2] Pérez-Tomás R. Multidrug resistance: retrospect and prospects in anti-cancer drug treatment [J]. Current medicinal chemistry, 2006, 13(16):1859-1876.

[3] Kuo M.T. Redox regulation of multidrug resistance in cancer chemotherapy: molecular mechanisms and therapeutic opportunities[J]. Antioxidants Redox signal, 2009, 11(1):99-133.

[4] Gillet JP, Gottesman MM. Mechanisms of multidrug resistance in cancer[J]. Methods in molecular biology, 2010, 596(1): 47-76.

[5] Espino PS, Drobic B, Dunn KL, et al. Histone modifications as a platform for cancer therapy[J]. J Cell Biochem, 2005, 94(6): 1088 1102.

[6] Chen K.G; Wang Y.C.; Schaner A.E.; Francisco B.; Durán G.E.; Juric D.; Huff L.M.; Padilla-Nash H.; Ried T.; Fojo T.; Sikic B.I. Genetic and epigencetic modeling of the origins of multidrug-resistant cells in human sacoma cell line[J]. Cancer Res, 2005, 65(20): 9388-9397.

[7] Townsend D.M; Manevich Y.; He L.; at al. Novel role for glutathione S-transferase pi. Regulator of protein S-Glutathionylation following oxidative and nitrosative stress [J]. Journal of biological chemistry, 2009, 284(1):436-445.

[8] Borst P.; Jonkers J.; Rottenberg S. What makes tumors multidrug resistant? [J]. Cell cycle, 2007, 6(22): 2782-2787.

[9] Kouzarides T. Chromatin modifications and their function[J]. Cell, 2007, 128(4): 693-705.

[10] Hung Xiaoping Xu Jiang. Histone Deacetylases Inhibitors cancer drug: Vorinostat[J]. Chemical Intermediat, 2009, (6):11-14.

[11] Liu T, Xu F, Du X, et al. Establishment and characterization of multi-drug resistance prostate carcinoma-initiationg stem-like cells from human prostate cancer cell lines 22RV1[J]. Mol Cell Biochem, 2010, 10.1007/s11010-010-0426-5.

[12] Peterson C.L.; Laniel M.A. Histones and histone modifications[J]. Curr Biol, 2004, 14(14): R546-551.

[13] Dokmanovic M.; Clarke C.; Marks P.A. Histone deacetylase in hibitors: overview and perpectives[J]. Mol Cancer Res, 2007, 5(10):981-989. 